\title{
Comercio bilateral entre China y América Latina antes de la pandemia. ¿Ahora qué?
}

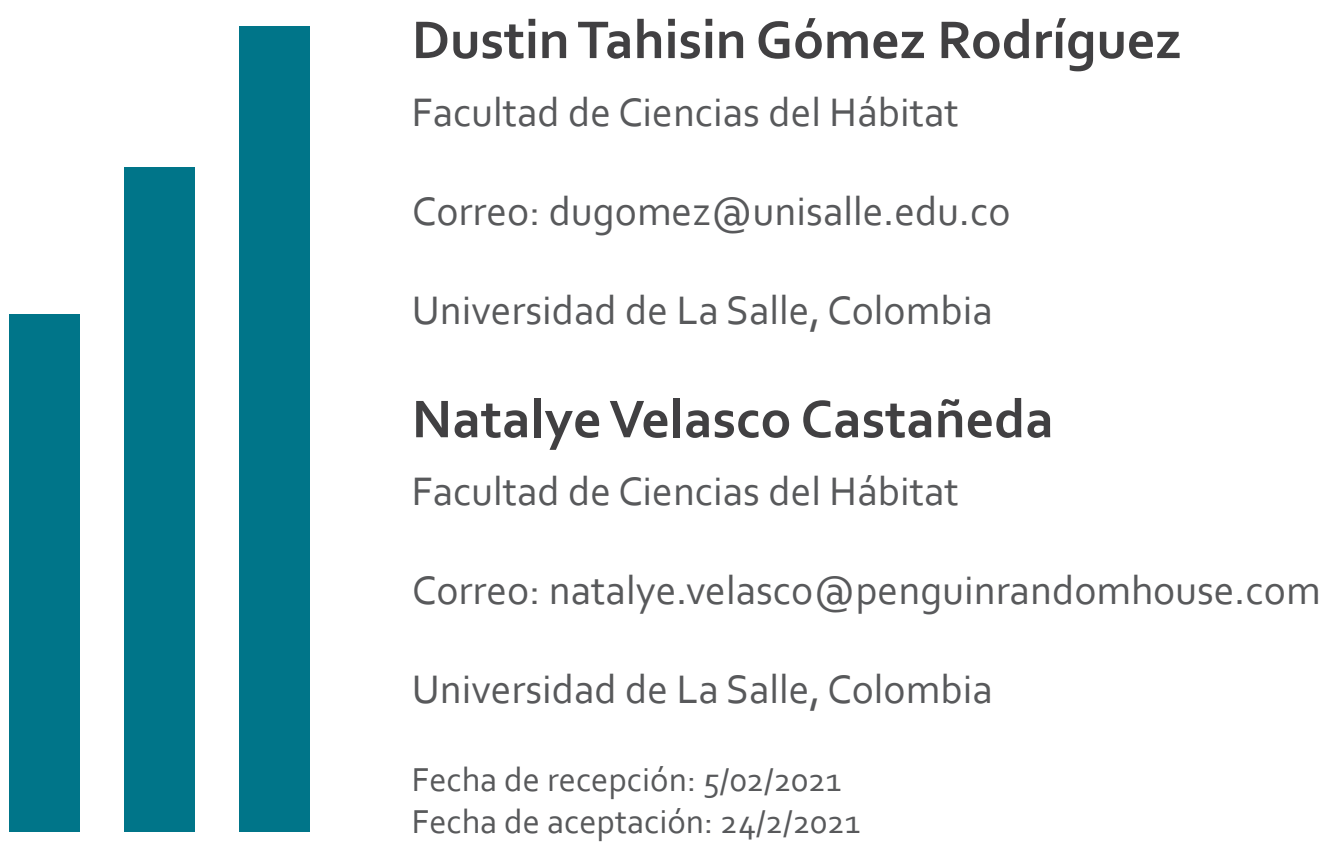

\section{Resumen}

Elobjetivo delartículoesrealizarunabrevedescripción del comercio bilateral entre EE. UU. y América Latina con China antes de la pandemia y cómo el nuevo orden que se avecina puede repercutir en los países latinoamericanos. La metodología es de corte cualitativo y el método es de revisión documental por matrices. La principal conclusión es que América Latina construye relaciones heterogéneas con China.
No obstante, se puede afirmar que el acercamiento entre ambos va en concordancia con el crecimiento económico impulsado por las exportaciones, el fomento a la inversión, así como una alternativa frente a los mercados internacionales de EE. UU. y la Unión Europea.

Palabras clave: comercio bilateral, cooperación, economía internacional, multilateral 


\title{
PRE-PANDEMIC BILATERAL TRADE BETWEEN CHINA AND LATIN AMERICA WHAT NOW?
}

\author{
Reflection paper
}

\begin{abstract}
The purpose of this paper is to present a brief description of pre-pandemic bilateral trade of the United States and Latin America with China, and to deliberate in what ways the upcoming new order could affect Latin American countries. Its methodology is qualitative based on document reviews by matrix. The main conclusion is that Latin America is building heterogeneous relations with China. This rapprochement between the two regions is consistent with economic growth fueled by exports and promotion of investments and constitutes an alternative to US and European Union markets in the sphere of international trade.
\end{abstract}

Keywords: bilateral trade, cooperation, international economy, multilateral 


\section{Introducción}

América Latina es un territorio con diferentes territorialidades, que histórica, geográfica, política y económicamente han soportado la presencia de diversas potencias extranjeras que se han y siguen aprovechándose de sus grandes recursos naturales, sociales a costos ambientales y humanos profundos en las diferentes sociedades que la componen (Gómez, 2021; Lo Brutto y Crivelli, 2016). Grosso modo, los países de este continente se han caracterizado por su precario y limitado crecimiento y desarrollo económico. Dando como resultado la pauperización de su capital humano y el aumento en la desigualdad social que ha incrementado la pobreza (Kalmanovitz, 2019; Bértola y Ocampo, 2012; Cepal, 2019).

No obstante, es un territorio que exhibe una enorme riqueza en recursos naturales: biodiversidad, agua dulce, ecosistemas, etc. De ahí, que sea un territorio «apetecido» por los intereses estratégicos tanto económicos como políticos de los EE. UU. y desde unas pocas décadas hacia el presente, por los cambios hegemónicos y geopolíticos provocados por China en el mundo (Xuetong, 2016; Boron, 2013; Lin, 2012; Bruckmann, 2012; Barrios ,2011; Estenssoro, 2010; Bruzzone, 2009; Fregapani, 2000).

Desde el inicio del siglo XXI, China ha emergido bajo una reconfiguración de la economía del mundo-capitalista. En particular, su influencia desde la cooperación económica y política, (aunque esta última menos) con varios gobiernos latinoamericanos de por medio en una relación «Cooperación Sur-Sur», no "Norte-Sur», como se ha presentado (Lo Brutto y Crivelli, 2019; Slipak y Ghiotto, 2019).

En efecto, y tomando como derrotero las líneas argumentativas de Lin y Wang (2017), la estrategia de Cooperación Sur-Sur antes mencionada que ha venido gestionando China es diferente a la lógica de Cooperación Norte-Sur. Precisamente, las dinámicas de la cooperación del gigante asiático han reflejado un nuevo modelo de colaboración que, no solamente se reduce a los acuerdos comerciales, sino que desarrolla una mixtura de inversiones, préstamos y créditos, lo que ha abierto la ventana a países con problemas financieros para poder acceder a los mercados internacionales con financiamiento (Dreher y Fuchs, 2012).

La necesidad China de productos primario-extractivos y de ahorro de Energía y agua virtual, como así también de diversificar las fuentes de insumos relevantes como el petróleo, hacen que la región (América Latina) resulte de interés para el país oriental. Al mismo tiempo, las asimetrías comerciales, financieras, 
tecnológicas y hasta militares entre los países de ALC y China, le permiten al último sacar provecho de dichas relaciones asimétricas, lógicamente con la anuencia de las elites locales que se enriquecen a partir de este vínculo (Slipak y Ghiotto, 2019, pp. 49-50).

En consecuencia, países latinoamericanos que cuestionaron fuertemente los Consensos de Washington y de Basilea (Gómez, 2015) han intensificado sus relaciones con China. En virtud de esto, surge la oportunidad de acceder a otros mercados con diferentes relaciones contractuales que son un posible nuevo orden mundial multipolar más equitativo (Bárcenas, Prado y Pérez, 2016). Del mismo modo, (Xuetong, 2016) se plantea que la reconfiguración de la hegemonía mundial se esboza en relación con la autoridad y el liderazgo internacional con el que se muestra China como objetivo para tratar de ampliar sus aliados en su proyecto de ascenso mundial. Sin embargo, Brukmann (2012) señala que, sin estrategias competitivas de los agentes económicos como la soberanía bajo una estrategia de largo plazo, los países latinoamericanos pueden tener menos oportunidades de hacer frente a las enormes presiones entre los grandes y su repartición del mundo: China y EE. UU.

En este sentido, el objetivo del artículo es realizar una breve descripción del comercio bilateral entre EE. UU. y América Latina con China antes de la pandemia y cómo el nuevo orden que posiblemente se avecina puede repercutir en los territorios de América Latina. De igual manera, la hipótesis del documento es que a medida en que pasa el tiempo, sobre todo los países con características parecidas políticamente con China profundizarán sus relaciones bilaterales en contraposición con EE. UU.

Para lograr el objetivo del presente artículo se divide en cuatro apartados. El primero inicia con la introducción ya expuesta. Continua con breves caracterizaciones del comercio bilateral teniendo como marco teórico los postulados de (Deltas, Desmet y Facchini, 2006; Alba, Jung y Park, 2010; De Santis, De Benedictis y Vicarelli, 2005; Kowalczyk y Wonnacott, 1992) y en la ventana de observación de la última década del siglo XXI antes de la pandemia entre el gigante asiático con EE. UU. y América Latina. Prosigue con un breve análisis para responder la pregunta problema del presente escrito que es la siguiente: ¿después de la pandemia, qué posibilidades hay entre China y América Latina?; finalmente, se exponen unas breves conclusiones del ejercicio investigativo. La metodología utilizada es cualitativa y el método es de revisión documental por medio de matrices que buscan identificar la divergencias y convergencias de los autores frente a las líneas argumentativas del artículo (Gómez, Ramos y Carranza, 2017; Gómez, Carranza y Camilo, 2016). 


\section{Comerio bilateral EE. UU. - China}

La literatura especializada tiende a resarcir las bondades del libre comercio internacional. No obstante, la evidencia ha demostrado que no necesariamente es así dado que las relaciones desiguales entre países, como conflictos e incluso guerras comerciales entre ellos acontecen entre las economías como por ejemplo la de China y EE. UU. Se podría afirmar que esta última se refiere al tipo de intercambio «ojo por ojo» en los aranceles entre estas dos economías (Macías, Chiatchoua y Lozano, 2020).

Decisivamente, en la primera década del año 2000, China y los Estados Unidos (EE. UU.) representan las dos economías más grandes del mundo desde la perspectiva del comercio internacional como de la inversión extranjera directa (IED) (Ornelas, 2018). El gigante asiático es el principal proveedor de importaciones de EE. UU., dejando a un lado, en segundo lugar, a su socio histórico, México, en el periodo 2000 y 2002 ( Cepal, 2011; Oviedo, 2014; Slipak y Ghiotto, 2019).

Desde el punto de vista del déficit comercial entre EE. UU. y China este incrementó en 419000 millones de dólares para el año 2018. De ahí que emergiera un conflicto comercial entre China y EE. UU. En efecto, EE. UU. ha incrementado sus aranceles en algunas importaciones provenientes de China, provocando que esta última haya respondido aumentado los aranceles de un subconjunto de importaciones de EE. UU. Este tipo de intercambios nombrados coloquialmente «ojo por ojo» en los aranceles son caracterizados como una «guerra comercial» (Estenssoro, 2019; FMI, 2019).

Retomando el FMI, para 2014 la economía China fue la mayor del mundo con 16.479 billones de dólares del PIB mundial ${ }^{1}$ frente al 16.277 de los EE. UU (Pardo, 2014). Para el año 2017, China tuvo como resultado un PIB total (PPA) mayor a los 23 billones de dólares. Continúa la Unión Europea con 19.9 y los EE.UU., en tercer lugar, con 19.3. No obstante, el Banco Mundial afirma que China en el año 2016 era la segunda economía con un PIB de 11.199 billones de dólares detrás de los EE. UU con 18.624 billones de dólares. Con el mismo propósito y siguiendo con los datos anteriores, el PIB total del mundo era de 75.872 billones de dólares. Donde China representaba el $14.7 \%$ de la economía del globo, mientras que los EE.UU., el 24.5\% (Banco Mundial, 2017; Central de Inteligencia Americana (CIA), 2017). Por lo tanto, los datos reflejan cómo la economía china superará a la estadounidense. Actualmente ocupa el segundo lugar en el mundo (Macías, Chiatchoua y Lozano, 2020; Ornelas, 2018).

1 Medición desde la Paridad de Poder Adquisitivo (PPA). 


\section{Comercio bilateral China - América Latina}

Los intercambios de bienes y servicios entre China y América latina se han basado en pragmatismo económico. Sobre todo, desde las reformas políticas del presidente Deng Xiaoping en el año 1978. Dicho pragmatismo se basa en categorías y conceptos marxistas, ya que estos argumentos conciben a la economía como base de la superestructura política (Xuetong, 2018). De ahí, que el interés trascendental del presidente actual, Xi Jinping, parte de una visión económica que destaca la relevancia del comercio bilateral, sin injerencia de los socios en las cuestiones políticas (Crievelli y Lo Brutto, 2019).

Precisamente desde el siglo $\mathrm{XXI}$, el gigante asiático se ha transformado en el segundo socio comercial de la región de América Latina y el Caribe, y el primero para países como Brasil, Chile y Perú. En particular, el comercio bilateral entre China y América Latina comenzó con 15.765 millones de dólares para el año 2001; en el año 2014 fue de 277.175 millones de dólares y, aunque hubo un decrecimiento, para el 2017 sobrepasaba los 100 mil millones de dólares, (Roldán, Castro, Pérez, Echavarría y Evan, 2016; Rosales y Kuwayama, 2012). Sin olvidar, que en el año 2015 se formalizó y se realizó el primer Foro China-CELAC (FCC), donde Xi Jinping indicó que el Estado de China aumentará su inversión en la región, en el periodo 20152025, la suma de 250.000 millones de dólares, algo muy alejado de las relaciones actuales entre EE. UU. y América Latina (Balderrama y Trejo, 2019; Rosales, 2019; LATINREPORTERS.com, 2015).

En virtud de los diálogos entre América Latina y China, en los años del 2001 al 2014, reflejó un hito histórico en la consolidación del comercio bilateral. Precisamente en datos, pasó de 15.765 millones de dólares en el 2001 a 277.175 millones de dólares en el 2014. Representando un crecimiento promedio de $26 \%$ anual, una cifra llamativa si se compara el comercio de América Latina con el mundo, el cual creció solamente $10 \%$ durante el mismo período de análisis (Roldan, et al., 2016). Desde el 2017 mostró recuperación ya que entre 2014-2017 decreció. Sin embargo, en el último año fue de un $16 \%$, acercándose al máximo histórico de 268.000 millones de dólares en el año 2013 (Crivelli y Lo Brutto, 2019; Cepal, 2018; Domínguez, 2018).

China ha establecido acuerdos con los países latinoamericanos en términos comerciales, de cooperación económica, política y cultural. Sus inversiones se han colocado principalmente en infraestructura, energía, manufactura, innovación tecnológica, agricultura y tecnología de la información. Según un informe del Foro Económico Mundial, China es el principal socio de Argentina, Brasil, Chile, Perú y Uruguay, y el segundo socio de México. Desde 2003 hasta 2017 se han invertido más de 110 mil millones de dólares y, aunque en los 
últimos años las inversiones chinas en la región han mermado (mientras las de EE. UU. siguen ocupando un rol protagónico) no dejan de ser significativas (García, 2018, p. 1).

Tomando otro periodo comparativo para enriquecer el análisis, desde el año 2005 hasta el 2017 se puede afirmar que creció el financiamiento multilateral y bilateral del gigante asiático. El cual se dirigió en primer lugar a la República Bolivariana de Venezuela, con 17 préstamos que ascienden a 62.200 millones de dólares. En segundo lugar, Brasil, que recibió préstamos por un monto de 42.100 millones de dólares. En tercer lugar, Argentina por un valor de 18.200 millones de dólares, seguido por el Estado de Ecuador con 17.400 millones de dólares y, por último, el Estado plurinacional de Bolivia por un monto de 3.500 millones de dólares. Sin embargo, el financiamiento por pare del Estado chino en América Latina se redujo en los últimos años con algo así de 9.000 millones de dólares porque en China, el Development Bank y el Eximbank redujeron los préstamos a Venezuela, principalmente por la coyuntura y la guerra comercial con EE. UU. (Crivelli y Lo Brutto, 2019; Myers y Gallagher, 2018; Cepal, 2018; Domínguez, 2017).

Sin olvidar que el comercio como la inversión y el financiamiento de China en América Latina ha sido en sectores relevantes que van en sintonía con las materias primas e hidrocarburos. Acompañados de conflictos socioambientales y socioeconómicos que han generado en algunos sectores críticas frente a este proceso (Macías, Chiatchoua y Lozano, 2020; Gallagher, 2016). No obstante, las posibilidades de intercambio más justo y en reciprocidad son variables que a medida que pase el tiempo se deberán mejorar, debido principalmente a las necesidades apremiantes del continente americano de diversificar sus mercados de conocimiento y sobre todo de valor agregado en sus bienes y servicios (Nieto y Rendón ,2019; Becerra, 2013). 


\section{Posibilidades de comercio bilateral entre China y Latinoamérica después de la pandemia}

En este momento se podría afirmar que existe una crisis civilizatoria o, mejor dicho, del orden establecido tanto económico como político (Maldonado, 2020 y Maldonado, 2019). En efecto, desde la segunda guerra mundial el mundo se ha regido por la potencia vencedora: EE. UU. (Stiglitz, 2020 y Stiglitz, 2012) Sin embargo, una nueva potencia está emergiendo y en consecuencia «el orden» antes mencionado cada vez es más exiguo o ha reflejado que las condiciones económicas y políticas promulgadas han sido desiguales entre EE. UU. y América Latina. Esto ha provocado que América Latina busque y abra la puerta a otros mercados como el que le presenta el país asiático: China.

Mientras China extiende el poder blando en todo el mundo para consolidar su crecimiento y desarrollo, Estados Unidos ejerce el poder duro en zonas específicas para lograr el control geopolítico, lejos de América Latina (Contardo, 2014, p. 71).

El gigante asiático promueve la multipolarización mundial la cual no está señalada por un único país. Precisamente lo que busca es la coordinación y la democratización en lugar del conflicto para salvaguardar la paz y el desarrollo mundial. Asimismo, busca una visión de seguridad desde la confianza recíproca, salvaguardando las diferentes sociedades que hay en el mundo. Por otra parte, promueve el desarrollo económico sustentable y equilibrado, en virtud de reducir la polarización de la riqueza, así como respetar y posicionar las directrices de la Organización de la Naciones Unidas, (Xiaoping, 2014). Desde esta óptica, los países latinoamericanos que fueron mencionados en los anteriores párrafos podrían establecer relaciones más amistosas y sobre todo con vínculos comerciales más horizontales, no condicionados políticamente (Contardo, 2014; Acemuglu y Robinson, 2013).

Por otro lado, desde la década pasada, China se ha convertido en mercado de exportación por excelencia de Brasil, Argentina, República Bolivariana de Venezuela, Cuba, Chile y Perú, contribuyendo en la diversificación del comercio bilateral. Sin embargo, han sido limitadas las exportaciones por parte de Ecuador y sobre todo por Centro América. Abriendo una posibilidad de mercado para estos países y en los primeros para ampliar la cuota exportadora. Asimismo, está el caso de Costa Rica, México y Salvador donde no solo exportan a China materia prima, sino que también exportan algunos productos manufacturados con alta tecnología e innovación reflejando el proceso de Investigación y Desarrollo (García, 2018; Bacchiega, 2014). 


\section{Conclusiones}

Las relaciones bilaterales entre América latina y China están permeadas inevitablemente por las relaciones del primero con EE. UU. Lo cual hace que China esté en franca competencia con EE. UU. En efecto, el aparato productivo de China en ascenso ha cambiado bastante la relación entre EE. UU. y América latina. De ahí, que se puede identificar una relación inversa, mientras el comercio bilateral es creciente entre China y América latina, con EE. UU. disminuye. Todo esto refleja que las condiciones que ostenta el gigante asiático van más en relación con las necesidades de América Latina, mientras que EE. UU. pretende mantener el «patio trasero».

La Cooperación Sur-Sur, promocionada por China, ha beneficiado un conjunto de países latinoamericanos, en virtud de que es una gran estrategia que revitaliza tanto la cooperación bilateral como multilateral en el plano mundial. De ahí, que los países en desarrollo, a juicio de los autores del presente escrito, deberían ampliar esta cooperación, dado que China promueve la dinamización y profundización de la Cooperación Sur-Sur, mientras que, hasta el momento, las relaciones Norte-Sur divulgadas por EE. UU. y la Unión Europea han contribuido a desequilibrios microeconómicos y macroeconómicos que han potencializado la ineficiencia en la distribución del ingreso en América Latina.

América Latina construye relaciones heterogéneas con China. No obstante, se puede afirmar que el acercamiento entre ambos va en concordancia con el crecimiento económico impulsado por las exportaciones, el fomento a la inversión, así como una alternativa frente a los mercados internacionales como los de EE. UU. y Europa, socios históricos que han decrecido. Acotando que América Latina en promedio exporta a China materias primas con algunas excepciones. En efecto, esto podría generar otra nueva Teoría de la Dependencia en términos de Raúl Prebisch, donde América Latina es el exportador de materia prima y China es el exportador de bienes y servicios con valor agregado. De ahí, que Latinoamérica tiene como desafío la incorporación de más conocimiento como contenido tecnológico a las exportaciones o sencillamente seguirá en las mismas. Es decir, antes exportaba materias primas a Europa y EE. UU. y ahora cambia por China. 


\section{Referencias}

Acemuglu, D., y Robinson J. (2013) Por qué fracasan los países: los orígenes del poder, la prosperidad y la pobreza. Ed. Ariel.

Alba, J., Jung, H. y Park, D. (2010). ¿Do Hub-and-Spoke Free Trade Agreements Increase Trade? A Panel Data Analysis. ADB Working Paper Series on Regional Economic Integration 46, 1-24.

Bacciega, J. (2014). Relaciones estratégicas de intercambio comercial y diplomático entre la República Popular China y América Latina. En Las relaciones entre China y América Latina. Ed Bogado, L. pp. 105-123. Editorial Universidad Nacional de la Plata. Buenos aires: Argentina. https://bit.ly/3aMowzG

Balderrama R., y Trejo, R. (2019). Hecho en China 2015 y la autosuficiencia en nuevas tecnologías. Revista de comercio exterior, 4, (19),53-67.

Banco Mundial , (2017). Banco Mundial mantiene proyección de crecimiento para China en 6,5\% este 2017.BM, Obtenido de : https://www.emol.com/noticias/ Economia/2017/04/13/854034/Banco-Mundial-mantiene-proyeccion-decrecimiento-para-China-en-65-este-2017.html

Banco Mundial. (2018). BM; PIB (US\$ a precios actuales).BM. https://datos. bancomundial.org/indicador/ny.gdp.mktp.cd

Barrios, M. (2011). Consejo latinoamericano de defensa: desafíos geopolíticos y perspectivas continentales. Biblos. Buenos Aires: Argentina.

Bárcenas, A., Prado, M., y Pérez, R. (2016). Relaciones económicas entre América latina y el Caribe y China Oportunidades y desafíos. Cepal. Santiago de Chile: Chile.

Becerra, L. (2013). Hacia un marco constructivista realista para el análisis de las relaciones internacionales desde las periferias. Colombia Internacional. 78: (236), 79-126.

Bértola, L., y Ocampo, J. (2012). El desarrollo económico de América Latina desde la independencia. FCE., México.

Boron, A. (2013). América Latina en la Geopolítica del Imperialismo. Caracas: Ministerio del Poder Popular para la Cultura Venezuela.

Bruzzone, E. (2009). Las Guerras del Agua. Buenos Aires: Capital Intelectual.

Bruckmann, M. (2012). Recursos Naturales y la Geopolítica de la Integración Sudamericana. Quito: Editorial IAEN. 
CIA. (2017). Country ComParíson: GDP (purchasing power pa-rity). CIA. https:// bit.ly/30AYtcN Cepal. (2019). Comisión Económica para América Latina y el Caribe. Panorama Social de América Latina 2019. Cepal. https://bit.ly/3AKC21a

Cepal. (2018). Comisión Económica para América Latina y el Caribe. Explorando nuevos espacios de cooperación entre América Latina y el Caribe y China., Santiago de Chile, Naciones Unidas.

Cepal. (2011). Comisión Económica para América Latina y el Caribe. La inversión extranjera directa en América Latina y el Caribe 2010.Documento informativo. Publicaciones ONU. Santiago de Chile: Chile.

Crivelli, E., y Lo Brutto, G. (2019). Las relaciones entre china y américa latina en la segunda década del siglo XXI. Cuadernos del CEL, VI :(7), 56-73.

Deltas, G., Desmet, K. y Facchini, G. (2006). Hub-and-Spoke Free Trade Areas. Discussion paper series, (5960), 1-33.

De Santis, R., De Benedictis, L. y Vicarelli, C. (2005). Hub-and-Spoke or Else? Free Trade Agreements in the 'Enlarged' European Union. The European Journal of Comparative Economics, ISAE Working Paper, 2(52), 245-26o.

Dreher, A., y Fuchs, A. (2012). Rogue aid? The determinants of China's aid allocation. Discussion Papers Courrant Research Center (93),1-48.

Domínguez, R. (2018). China y la construcción de un régimen internacional de Cooperación Sur-Sur. Carta Internacional .13: (1), 38-72.

Domínguez, R. (2017). La Princesa y el Dragón: Cooperación China en América Latina y más allá. Revista Internacional de Cooperación y Desarrollo 2: (4), 3-27.

Estenssoro, J. (2019). América Latina frente a la creciente tensión entre China Y EE.UU. ¿Hacia Dónde va el Mundo? Revista do servicio público 5(1):43-58.

Estenssoro, J. (2010). Crisis ambiental y cambio climático en la política global: un tema crecientemente complejo para América Latina. Universum, 25: (2),57-77.

Fregapani, G. (2000). Amazónia: A Grande Cobija Internacional. Brasilia: Thesaurus.

FMI (2019). Informes de Perspectivas de la Economía Mundial. Fondo Monetario Internacional. https://bit.ly/3jdU7yy

Gallagher, K., (2016). The China triangle: Latin America's China boom and the fate of the Washington consensus, Oxford University Press.

García, A. (2018). China en América Latina, según Estados Unidos. CELAG. https:// www.celag.org/china-america-latina-segun-eeuu/

Gómez, D. (2021). Sostenibilidad. Inclusión y Desarrollo, 8(1), 131-143. 
Gómez, D., Ramos, C., yCarranza, Y. (2017). Aportes de negociadores internacionales para el crecimiento, la innovación, el emprendimiento y la competitividad de las empresas colombianas. FACCEA, 7(2), 157-164.

Gómez, D., Carranza, Y., y Camilo, R. (2016). Revisión documental, una herramienta para el mejoramiento de las competencias de lectura y escritura en estudiantes universitarios. Chakiñan (1), 46-56. https://doi.org/10.37135/chk.002.01.04

Gómez, D. (2015). Introducción al Neoliberalismo y la educación. En Neoliberalismo y Educación. Ed Gómez, D. Editorial Corporación Unificada de Educación Superior. Bogotá: Colombia.

Kalmanovitz, S. (2019). Nueva historia económica de Colombia. Editorial Penguin Random House, Bogotá: Colombia.

Kowalczyk, C. y Wonnacott, R.J. (1992). Hubs and Spokes, and Free Trade in the Americas. NBER Working Paper, (4198), 1-33.

LATINREPORTER. (2015). China quiere invertir en América Latina 250.000 millones de dólares en 10 años. Latinreporters.com. Madrid, 10 de enero de 2015. https:// bit.ly/3AOoCke

Lin, J., y Wang, Y. (2017). Going Beyond Aid, Development, Cooperation for Structural Transformation, United Kingdom. Cambridge Press University.

Lin, J. (2012)., New Structural Economics, A Framework for Rethinking Development and Policy, Washington D.C. Banco Mundial.

Lo Brutto, G., y Crivelli, E. (2016). La Unión de Naciones Sudamericanas (UNASUR) en la disputa por una integración regional en América Latina. Revista Española de Desarrollo y Cooperación, 37: (6), 107-115.

Macías, J., Chiatchoua, C., y Lozano, M. (2020). Análisis del conflicto comercial Estados Unidos-China: Un modelo de Richardson difuso. Revista Latinoamericana De Investigación Social, 2(3), 16-34.

Maldonado, C. (2020). Occidente, la civilización que nació enferma. Editorial Desde Abajo. Bogotá: Colombia.

Maldonado, C. (2019). Turbulencias. Sobre ciencias y otras complejidades. Tomo 1. Editorial Universidad del Bosque. Bogotá: Colombia.

Ministerio de Relaciones Exteriores de la República Popularde China (2008). Texto íntegro del Documento sobre la Política de China hacia América Latina y el Caribe. MRERPC. https://bit.ly/2XmmKSx

Myers M., y Gallagher, K. (2018). Chinese development finance «down but not out» in 
Latin Americ. The Global Americans, 30 de marzo de 2018. https://bit.ly/3non5Dq Nieto, P., y Rendón, J. (2019). ¿Es la integración regional un vehículo para la convergencia? El caso de Mercosur 1990-2014. Revista Finanzas y Política Económica 11:(1),23-40.

Ornelas, R. (2018). La competencia entre Estados Unidos y China por el liderazgo mundial: Un panorama desde las inversiones extranjeras. México y la Cuenca del Pacífico, 7(21):53-93. https://doi.org/10.32870/mycp.v7i21.552

Pardo, P. (2015). The IMF declares China the largest economy in the world. The World, Washington, 10/8/2014. Web. June 7, 2015. Retrieved at. https://www. elmundo.es/economia/2014/10/08/54354d27268ezeeg448b4581.html

Oviedo, E. (2014). América Latina: entre la hegemonía estadounidense y la influencia china, Ponencia en Flacso-ISA, Joint International Conference. Global and Regional Powers in a Changing World.

Roldán, A., Castro, A., Pérez, C., Echavarría, P., y Evan, R. (2016). La presencia de China en América Latina. Comercio, inversión y cooperación económica. Colombia: Fundación Konrad Adenauer.

Rosales, O. (2019). El conflicto económico Estados Unidos- China. Economía y Administración, (173), 11-14. https://bit.ly/3AOHm3x

Rosales, O., y Kuwayama, M. (2012). China y América Latina y el Caribe. Hacia una relación económica y comercial estratégica. CEPAL, Santiago de Chile: Chile.

Slipak, A., y Ghiotto, L. (2019). América latina en la nueva ruta del sol. CUADERNOS DEL CEL. IV, (7), 26-55.

Stiglitz, J. (2020). Capitalismo progresista. La respuesta a la era del malestar. Taurus, Barcelona: España.

Stiglitz, J. (2012). El precio de la desigualdad. Taurus. Barcelona: España.

Xiaoping, S., Contardo, M., Ricardo, L. y Bachhiega, J. (2014)). Las relaciones entre China y América Latina y el Caribe. La visión estratégica del país asiático y el declive del liderazgo norteamericano en la región. En Las relaciones entre China y América Latina. Ed Bogado, L. pp. 57-75 Editorial Universidad Nacional de la Plata. Buenos aires: Argentina. http://www.iri.edu.ar/images/Documentos/ publicaciones/relaciones_china_alatina.pdf

Xuetong, Y. (2018). Chinese Values vs. Liberalism: ¿What Ideology Will Shape the International Normative Order?, The Chinese Journal of International Politics .11: (1) 1-22.

Xuetong, Y. (2016). Political Leadership and Power Redistribution. Chinese Journal of International Politics, 9: (1), 1-26. 